\title{
Estimación espacial de la evapotranspiración mediante imágenes de satélite Landsat y el modelo SEBAL en el humedal Paraíso - Huacho
}

\section{Spatial estimation of evapotranspiration by Landsat satellite images and the SEBAL model in the Paraíso wetland - Huacho}

\author{
Erick Garcia ${ }^{1}$ y Miguel Ángel Lleellish ${ }^{2}$ \\ ${ }^{1}$ Autoridad Nacional del Agua (ANA), Calle diecisiete $N^{\circ} 355$, Urb. El Palomar - San Isidro Lima, Perú. \\ ${ }^{2}$ Ministerio del Ambiente (MINAM), Calle Los Nogales N² 236, San Isidro Lima, Perú.
}

\section{RESUMEN}

Los humedales están entre los ecosistemas más valiosos del planeta porque albergan una gran biodiversidad. Además, brindan muchas funciones relacionada con los recursos hídricos, como ser fuentes naturales de agua, reguladores del ciclo hidrológico y el clima, zonas de descarga y recarga de acuíferos, barreras naturales contra las inundaciones y la intrusión marina en los acuíferos costeros, entre otras. El conocimiento de las demandas ecológicas de agua en los humedales es de vital importancia para incluir a estos ecosistemas en una gestión integrada de los recursos hídricos. En este estudio, se presentan los resultados obtenidos en la estimación de la evapotranspiración en el humedal Paraíso de Huacho, mediante la aplicación del modelo de balance de energía superficial conocido como SEBAL a una subescena del satélite Landsat 7 ETM+ con fecha 04 de abril de 2000, la cual coincide con el inicio de la época de afloramiento en el humedal. La estimación de la evapotranspiración actual $\left(\mathrm{ET}_{\mathrm{a}}\right)$ estuvo en el rango de 0-6.3 mm/día, con los valores más bajos en las zonas desérticas y las parcelas en descanso, y los valores más altos en los espejos de agua y la vegetación del humedal. El humedal evapotranspira en altas tasas, con una $\mathrm{ET}_{\text {a }}$ máxima de $4.9 \mathrm{~mm} /$ día y un valor medio de $4.6 \mathrm{~mm} /$ día para la fecha analizada. La evapotranspiración de referencia (ETo) estimada mediante el método FAO Penman-Monteith, para la fecha de paso del satélite, fue de $3.9 \mathrm{~mm} / \mathrm{día}$ y con la inclusión del coeficiente del cultivo $(\mathrm{Kc})$ dio valores de 4.2 y $4.3 \mathrm{~mm} /$ día, para la vegetación del humedal y para el cultivo de alfalfa respectivamente. Estos dos valores están cerca a la $\mathrm{ET}_{\mathrm{a}}$ de 4.6 y $3.9 \mathrm{~mm} /$ día estimada por SEBAL para la misma vegetación y que se encuentra en condiciones optimas de crecimiento. Los resultados obtenidos demuestran que es factible estimar la $\mathrm{ET}_{a}$ mediante imágenes de satélite con un error medio relativo menor al $10 \%$.

Descriptores: teledetección, sensoramiento remoto, evapotranspiración, humedales, SEBAL.

\section{ABSTRACT}

Wetlands are among the most valuables ecosystems in the world, because support a great biodiversity. Besides, provides many hydrological functions such as surface water sources, regulation of hydrological cycle, weather and aquifer recharge and discharge zones, they are also natural barriers to flooding and sea intrusion in coastal aquifer. The knowledge of the environmental water demands for wetlands is of vital importance to include these ecosystems in water resources management. In this study, the results obtained in the evapotranspiration estimation in the Paraíso wetland are presented. The results were obtained by the application of the surface energy balance model known as SEBAL over a sub scene of Landsat ETM+ satellite dated April 8, 2000, this coincide with the beginning of upwelling season in the wetland are presented. Actual evapotranspiration $\left(E T_{a}\right.$ ) estimates was in the range of $0-6.3 \mathrm{~mm} /$ day, the lowest values were in desert and fallow land areas and the highest values were in surface water bodies and wetland vegetations. The wetland evapotranspiration rate was high, with a maximum ET of $4.9 \mathrm{~mm} /$ day and average value of $4.6 \mathrm{~mm} /$ day. The reference evapotransporation ( $\mathrm{ET}_{\mathrm{o}}$ ) estimated in the satellite overpass date was $3.9 \mathrm{~mm} /$ day and adjusted with the crop coefficient $(\mathrm{Kc})$ was 4.2 y $4.3 \mathrm{~mm} /$ day, for wetland vegetation and alfalfa crops. This values area 
close to the $\mathrm{ET}_{\mathrm{a}}$ of 4.6 y $3.9 \mathrm{~mm} /$ day estimated by SEBAL for the same vegetation in healthy conditions. The results obtained shows that are feasible to estimate the $\mathrm{ET}_{a}$ by satellite images with a relative mean error lower than $10 \%$.

Keywords: remote sensing, evapotranspiration, wetlands, SEBAL.

\section{INTRODUCCIÓN}

Los humedales que existen en la región costera del Perú son dependientes del aporte de las aguas subterráneas y en una menor medida de los flujos de aguas superficiales. Ante la demanda creciente de la extracción del agua subterránea, principalmente con fines agrícolas y productivos, estos ecosistemas se ven constantemente amenazados ante una posible disminución de este recurso. La principal dificultad para conservar estos ecosistemas desde un punto de vista ecohidrológico es la falta de información hidrometeorológica medida in situ, indispensable para estimar el consumo de agua en estos ecosistemas. El uso de las imágenes de satélite en combinación con información meteorológica y los modelo de balance de energía en superficie, se muestran actualmente como una herramienta alternativa y complementaria a las técnicas tradicionales para estimar la evapotranspiración o consumo del agua, de tal manera que esta información contribuya a la conservación de estos ecosistemas.

La evapotranspiración ha sido observada por décadas, sin embargo, un mejor entendimiento de su distribución espacial y a diferentes escalas es posible actualmente por medio de las imágenes de satélite. Generalmente los métodos para estimar la evapotranspiración han sido agrupados en cuatro categorías (Ahmad et al., 2005), ej., los métodos hidrológicos (balance de agua), mediciones directas (lisímetros), métodos micro-meteorológicos (balance de energía) y métodos empíricos o métodos combinados, basados en el balance de energía o factores climáticos (Thomthwaite y Mather, 1955). La mayoría de estos métodos sólo pueden proveer estimaciones puntuales de la evapotranspiración, lo cual muchas veces no es suficiente para capturar la variación espacial en grandes áreas. Por otra parte los modelos hidrológicos pueden hacer estimaciones de los patrones de la evapotranspiración, pero requieren una enorme cantidad de datos medidos en campo, lo cual a menudo no está disponible en países en desarrollo y menos en zonas de humedales por su difícil accesibilidad.

Durante las últimas décadas, muchos estudios han sido hechos por investigadores para estimar los flujos de energía y los intercambios que ocurren en superficies con vegetación y sin vegetación, usando modelos basados en sensores remotos. Estos flujos de energía han sido estudiados en diversas cubiertas del suelo como campos agrícolas (Kustas, 1990; Bastiaanssen, 2000) y humedales (Mohamed et al., 2004; y Oberg y Melesse, 2006).

Entre los métodos más empleados para estimar la evapotranspiración mediante el uso de imágenes de satélite y la ecuación del balance de energía, está SEBAL (Surface Energy Balance Algorithm for Land) Bastiaanssen et al. (1998a), donde la evapotranspiración actual es estimada como un residual del balance de energía. SEBAL ha sido validado a diferentes niveles como un eficiente $y$ efectivo método para cuantificar la evapotranspiración a nivel de cuenca y sub-cuenca (Allen et al. 2001). Además Bastiaanssen et al. (1998b, 2000), y Tasumi et al. (2000) obtuvieron resultados concordantes en regiones semiáridas. Hafeez et al. (2002), y Morse et al. (2000) lo hicieron en regiones tropicales, de sabanas y montañosas, respectivamente. Allen et al. (2001) encontró calibraciones satisfactorias mediante el uso de lisímetros de campo.

En este trabajo se ha aplicado SEBAL, con el objetivo de evaluar la factibilidad de estimar la evapotranspiración actual en el humedal Paraíso, considerando la fecha de adquisición de la imagen de satélite.

\section{METODOLOGÍA}

\section{Área de Estudio}

El humedal Paraíso, forma parte de los humedales de la costa central del Perú. Se encuentra ubicado en la Provincia de Huaura, Región Lima, entre los $77^{\circ} 36^{\prime} \mathrm{O}$ y $-77^{\circ} 33^{\prime} \mathrm{O}$, y los $-11^{\circ} 9^{\prime} \mathrm{S}$ y los $-11^{\circ} 13^{\prime} \mathrm{S}$. Tiene una extensión aproximada de 638 ha, las cuales incluyen dos espejos de aguas conocidas como laguna sur (186.5 ha) y laguna norte (34.7 ha), la primera con una mayor profundidad $(1.5 \mathrm{~m})$ a diferencia de la segunda $(0.5 \mathrm{~m})$. A su vez, el humedal comprende diferentes tipos de vegetación propias de estos ecosistemas hidromórficos (totorales, juncales y gramadales).

El clima de la zona de estudio, según la clasificación climática de Thornthwaite (SENAMHI, 1988) 
ECIPERÚ

corresponde a un clima árido, con escasa precipitación en todas las estaciones, normalmente

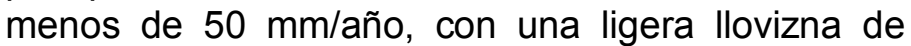
junio a setiembre, semi-cálido con una temperatura regularmente estable en torno a los $19^{\circ} \mathrm{C}$ y con alta humedad a excepción de los meses de verano.

Según la nueva delimitación oficial de unidades hidrográficas para el país adoptando el método de Pfafstetter (MINAG, 2008), el humedal Paraíso se encuentra ubicado en la unidad hidrográfica 137559 de tipo intercuenca. Limita por el norte con la cuenca del rio Huaura, por el sur con la cuenca del rio Chancay-Huaral y por el oeste con el océano pacifico (Fig.1). La administración de los recursos hídricos le corresponde a la Administración Local de Agua Huaral-Huaura.

El humedal Paraíso debe su origen a la inundación sucedida en el mes de marzo del año 1973, como consecuencia del rebose de los excedentes hídrico de la irrigación Santa Rosa. Estas aguas antes de seguir su curso natural hacia el mar y debido a la topografía de la zona, se depositaron en depresiones del terreno, dando origen a una albufera que por un par de meses fue una sola, hasta que los materiales de arrastre de un médano derruido cegaron la parte central, convirtiéndola en un extenso pantano y dividiéndola en dos albuferas, norte y sur como es en la actualidad (Figura 1).

Figura 1: Ubicación del humedal Paraíso - Huacho

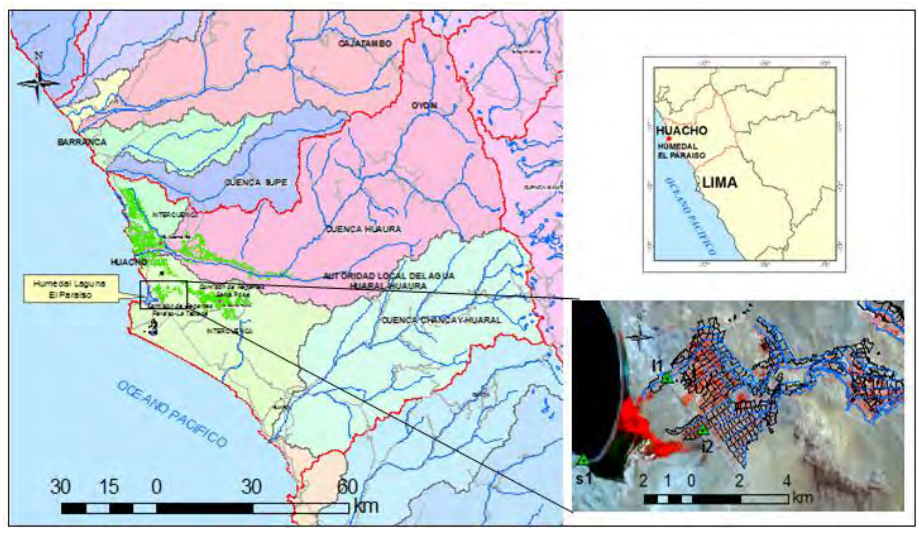

\section{Materiales}

Se empleó una sub-escena del satélite Landsat ETM (Path 006 y Row 068) de fecha 08 de abril de 2000, descargada gratuitamente de la Interface de Datos para las Ciencias de la Tierra (ESDI) de la Universidad de Maryland.

En la Tabla1, se presenta la información meteorológica usada. Todas las variables meteorológicas fueron tomadas de la estación Alcantarilla, excepto el dato de la velocidad del viento, que al no contarse con medidas para la fecha de la imagen en dicha estación, éste se tomó de la estación Santa Rosa. Ambas estaciones son del Servicio Nacional de Meteorología e Hidrología (SENAMHI).

\begin{tabular}{|l|l|l|l|l|l|}
\hline $\begin{array}{l}\text { Fecha } \\
\text { 08/04/2000 }\end{array}$ & $\begin{array}{l}\text { Temp. } \\
\text { Max. } \\
\left({ }^{\circ} \mathrm{C}\right)\end{array}$ & $\begin{array}{l}\text { Temp. } \\
\text { Min. } \\
\left({ }^{\circ} \mathrm{C}\right)\end{array}$ & $\begin{array}{l}\text { Horas } \\
\text { de sol } \\
(\mathbf{h r s} .)\end{array}$ & $\begin{array}{l}\text { Veloc. } \\
\text { viento } \\
(\mathbf{m} / \mathbf{s})^{1}\end{array}$ & $\begin{array}{l}\text { Humed. } \\
\text { Relativa } \\
(\%)\end{array}$ \\
\hline $\begin{array}{l}\text { Estación } \\
\text { Alcantarilla }\end{array}$ & 26 & 17.4 & 8.3 & 3 & 80.3 \\
\hline
\end{tabular}

Tabla 1: Información meteorológica necesaria para la aplicación de SEBAL.

\section{Modelo SEBAL}

El modelo SEBAL calcula los componentes del balance de energía combinando parametrizaciónes físicas y relaciones empíricas con una mínima cantidad de información de campo (ecuación 1). La información clave para aplicar este modelo, consiste de la radiancia espectral medida por los satélites en las regiones del visible, infrarrojo cercano e infrarrojo térmico del espectro electromagnético e información meteorológica de rutina como la velocidad del viento, temperatura del aire, radiación solar y humedad del aire.

$$
R_{n}=G+H+\lambda E
$$

Donde: $\mathrm{R}_{\mathrm{n}}$ es la radiación neta $\left(\mathrm{W} / \mathrm{m}^{2}\right)$, $\mathrm{G}$ el flujo de calor en el suelo $\left(\mathrm{W} / \mathrm{m}^{2}\right)$,H el flujo de calor sensible $\left(\mathrm{W} / \mathrm{m}^{2}\right)$ y $\lambda \mathrm{E}$ es el flujo de calor latente $\left(\mathrm{W} / \mathrm{m}^{2}\right)$.

En SEBAL, la radiación neta $\left(\mathrm{R}_{\mathrm{n}}\right)$ es computada mediante la información de la radiancia espectral en las diferentes longitudes del espectro captada por los satélites de observación terrestre. Sólo podrán usarse aquellos satélites que cuenten con una banda en la región del infrarrojo térmico, la cual servirá para estimar la temperatura de superficie. El Flujo de calor en el suelo ( $G$ ) es estimado a través 
de la radiación neta, la temperatura de superficie y el cálculo de algunos índices de vegetación. El flujo de calor sensible $(\mathrm{H})$ se ajusta mediante la selección de dos pixeles anclas tomados de la imagen, que representan condiciones extremas, uno de aridez (flujo de calor latente cercano a cero) y el otro de humedad (flujo de calor sensible cercano a cero). Con estos tres componentes se procede a calcular el flujo de calor latente $(\lambda \mathrm{E})$, como un residual del balance de energía para el momento de paso del satélite. Para la conversión de los valores instantáneo del $\lambda \mathrm{E}$ a valores diarios (24horas) se usa la fracción evaporatíva y la radiación neta diaria ( $\left.R n_{24}\right)$, (Ecuación 2 y 3 ).

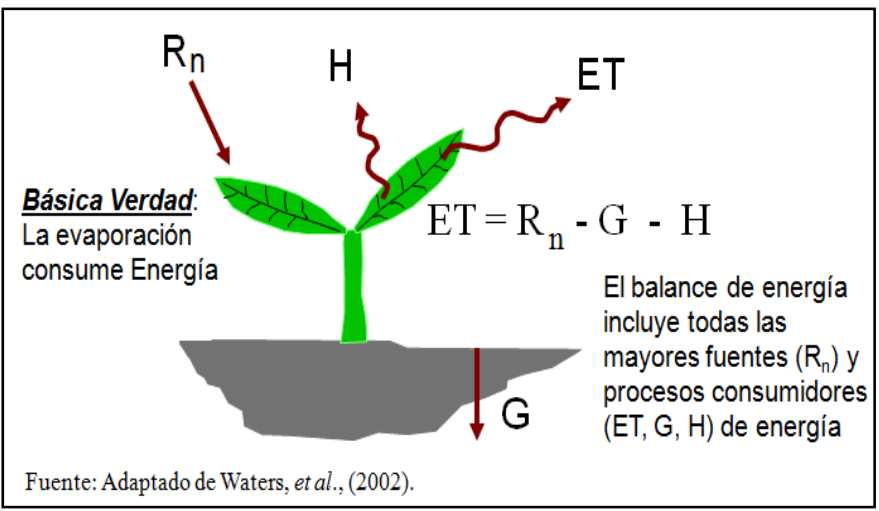

En la fig. 2 se puede ver un esquema de los componentes del balance de energia. Un mayor detalle de las ecuaciones que son usadas en SEBAL, pueden encontrarse en (Waters et al., 2002).

$$
\begin{gathered}
\Lambda=\frac{\lambda E}{R_{n}-G}=\frac{R_{n}-G-H}{R_{n}-G} \\
\lambda E_{24}=\Lambda \times R n_{24}
\end{gathered}
$$

Figura 2: Componentes del balance de energía en el modelo SEBAL

El cálculo de la evapotranspiración usando SEBAL fue realizado en el programa ERDAS IMAGINE, mediante la programacion de las ecuaciones en el lenguage de macros de ERDAS (EML). Para el análisis posterior de los mapas de evapotranspiración generados y su integración con otras capas de información se usó el programa de Sistemas de Información Geográfica ArcGis. El mapa de evapotranspiracion calculado por SEBAL se puede ver en la Figura 3.

\section{RESULTADOS Y DISCUSIÓN}

\section{Métodos de Validación}

Debido a que no existen datos de evapotranspiración medidos en el humedal, se procedió a validar el mapa de evapotranspiración calculado por SEBAL con dos métodos alternativos. El primero asumiendo que la vegetación perenne y permanentemente húmeda del humedal, debería de evapotranspirar valores cercanos a la evapotranspiración de referencia $\left(E T_{r}\right)$ (Jia et al., 2009). En el humedal Paraíso, la superficie ideal podría ser la vega de ciperáceas, aunque las condiciones de humedad en el humedal pueden variar con el tiempo, según los aportes de las aguas subterráneas y superficiales, en la vega de ciperáceas la vegetación y el suelo se mantienen permanentemente húmedos.

Para la selección de los pixeles que presentan una vegetación permanentemente húmeda, se usó el mapa de cobertura y usos del suelo, y las imágenes de temperatura de superficie y albedo estimados con SEBAL, ya que los pixeles húmedos presenta un valor bajo de temperatura y albedo.

En la vega de ciperáceas se obtuvo una $\mathrm{ET}_{\mathrm{a}}$ media de $4.6 \mathrm{~mm} /$ día. La $\mathrm{ET}_{\text {o }}$ estimada por el método $\mathrm{FAO}$ Penman-Monteith para la fecha de toma de la imagen fue de $3.9 \mathrm{~mm} / \mathrm{día}$. Dado que la evapotranspiración estimada por el modelo SEBAL viene a ser la evapotranspiración actual. Es necesario multiplicar el valor de la $\mathrm{ET}_{0}$ por un coeficiente del cultivo (Kc), el cual es dependiente del estado fenológico de la planta (Ecuación 4). En el estudio de la FAO (Allen et al., 1998) se consigna un valor del coeficiente de cultivo (Kc) en climas templados para la vegetación de humedales de 1.2 (etapa media de crecimiento). Este valor fue corregido para las condiciones climáticas de la zona, obteniéndose un $\mathrm{kc}$ de 1.08 y una evapotranspiración del cultivo $\left(\mathrm{ET}_{\mathrm{c}}\right)$ de $4.2 \mathrm{~mm} /$ día. Este valor calculado se acerca a la $\mathrm{ET}_{\mathrm{a}}$ estimada por SEBAL, con una diferencia de $9.5 \%$ entre ambas (Tabla 2).

En el segundo método, se empleó la cedula de cultivo para el valle de Huaura, específicamente para la comisión de regantes La Tablada-Paraíso, junto con la cartografía de parcelas del valle elaborada por el PETT (1/10000), cuya base de datos cuenta con información de los cultivos que normalmente son sembrados por los agricultores. Con esta información se procedió a identificar las parcelas que siembran alfalfa en el mapa de evapotranspiración calculado por SEBAL, obteniendo un valor medio de 
ECIPERÚ

$3.9 \mathrm{~mm} /$ día. Para el caso de la alfalfa se tomó un Kc de 1.10, consignado para el mes de abril, en el estudio hidrológico elaborado para el valle de Huaura (INRENA, 2004). Con este valor se calculó la $E T_{c}$ que fue de $4.3 \mathrm{~mm} /$ día. Este valor calculado se acerca a la $\mathrm{ET}_{\mathrm{a}}$ estimada por SEBAL, con una diferencia de $9.3 \%$ entre ambas (Tabla 2 ).

$$
E T_{c}=E T o \times K c
$$

La Tabla 2 muestra los resultados obtenidos en las comparaciones efectuadas, con los dos métodos alternativos de validación. Los resultados obtenidos muestra la efectividad del método SEBAL, obteniéndose discrepancia del $9.5 \%$ para la vegetación de los humedales con respecto a la $\mathrm{ET}_{\mathrm{c}} \mathrm{y}$ de $9.3 \%$ para la alfalfa con respecto a la $\mathrm{ET}_{\mathrm{c}}$.

Tabla 2: Comparación de los valores de la evapotranspiración estimados por SEBAL y el método Penman-Monteith (FAO56) con la inclusión del coeficiente del cultivo (Kc).

\begin{tabular}{|c|c|c|c|}
\hline $\begin{array}{c}\text { Cobertura } \\
\text { del suelo }\end{array}$ & $\begin{array}{c}\mathrm{ET}_{\text {aSEBAL }} \\
\text { (mm/día) }\end{array}$ & $\begin{array}{c}\mathrm{ET}_{\mathbf{c}} \\
(\mathbf{m m} / \text { día) }\end{array}$ & $\begin{array}{c}\text { Error medio } \\
\mathbf{( \% )}\end{array}$ \\
\hline $\begin{array}{c}\text { Vega de } \\
\text { ciperáceas }\end{array}$ & 4.6 & 4.2 & 9.5 \\
\hline Alfalfa & 3.9 & 4.3 & 9.3 \\
\hline
\end{tabular}

\section{Evaluación Espacial de la $\mathrm{ET}_{\mathrm{a}}$ Diaria}

El mapa de evapotranspiración estimado en base a la imagen Landsat y el modelo SEBAL para el día 08 de abril de 2000, en el ámbito conformado por el humedal Paraíso y el bloque de riego Paraíso-La Tablada, se muestra en la Fig. 3. En la Tabla 3, se muestran los valores de la $\mathrm{ET}_{\mathrm{a}}$ extraídos para las dos clases predominantes de cobertura del suelo en el humedal.

El rango de la $E T_{a}$ se encuentra entre 0 a 6.3 $\mathrm{mm} /$ día. Los valores de $\mathrm{ET}_{\mathrm{a}}$ para los espejos de agua del humedal están en el rango de 4.3 a 6.1 $\mathrm{mm} / \mathrm{día}$, con un valor medio de $5.2 \mathrm{~mm} /$ día. El valor total de la evaporación en los espejos de agua del humedal, los que en conjunto tienen una extensión de 221.2.6 ha, fue estimado en $11,502 \mathrm{~m}^{3}$, valor que representa el volumen de agua perdido por evaporación para el día considerado. Debido a que los cuerpos de agua del humedal son de escaza profundidad, se consideró en el modelo SEBAL que sólo un valor de $3 \%$ de la radiación neta pasa a formar parte de la reserva calorífica de los espejos de agua. En los espejos de agua de mayor profundidad, la reserva calorífica es mayor, además esta energía almacenada en el agua no está disponible de manera inmediata para la evaporación.

Los valores de la $E T_{a}$ para la vegetación del humedal están en el rango de 3.4 a $4.9 \mathrm{~mm} /$ día, con un valor medio de $4.6 \mathrm{~mm} /$ día. Esta representa la $\mathrm{ET}_{\mathrm{a}}$ que ocurre en la vegetación conformada principalmente por junco, grama y algunos parches de totora, que con una extensión de 384.1 ha, evapotranspiran $17,669 \mathrm{~m}^{3}$ de agua provenientes principalmente de las filtraciones del acuífero. La evapotranspiración en la vegetación del humedal es alta $(4.6 \mathrm{~mm} /$ día $)$, debido a la vegetación herbácea perenne que crece en el humedal, y a que el agua siempre está disponible para satisfacer la demanda evaporatíva de la atmosfera durante todo el año, especialmente en los meses de verano donde la nubosidad es baja.

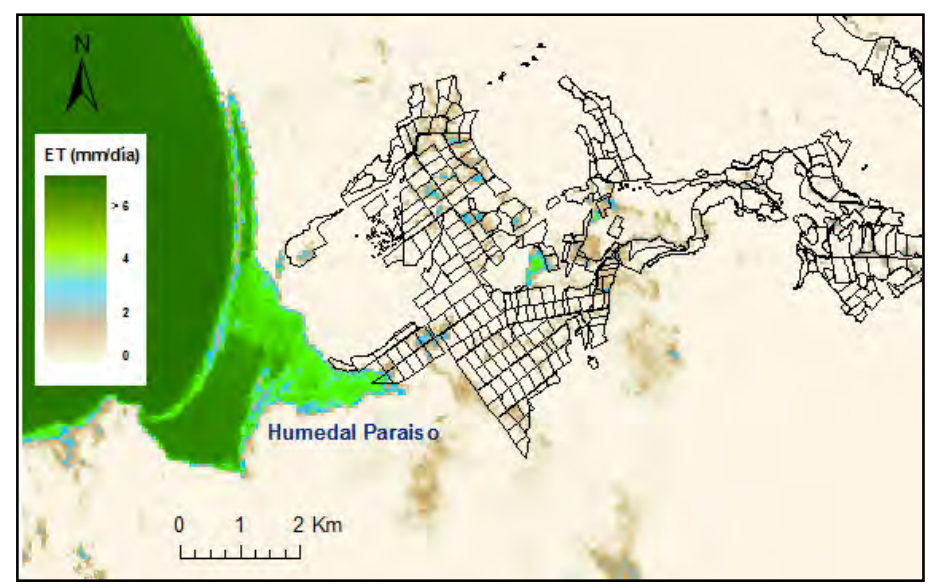

Figura 3: Mapa de la $\mathrm{ET}_{\mathrm{a}}$ estimada el 08/04/2000 por SEBAL en el humedal Paraíso.

Los valores de $\mathrm{ET}_{\mathrm{a}}$ estimados para las áreas agrícolas de la comisión de regantes La TabladaParaíso, están en el rango de 0 (suelo en descanso) a $3.9 \mathrm{~mm} /$ día en las parcelas con cultivos que presentan un alto vigor vegetal al momento de paso del satélite, coincidiendo con la $\mathrm{ET}_{\text {o }}$ estimada con la información meteorológica. Aunque la campaña agrícola se inicia en el mes de agosto en el valle de Huaura, el cultivo de la alfalfa es permanente y ocupa la mayor extensión de los cultivos en la comisión de regantes La Tablada-Paraíso, cerca de 247 ha para la campaña agrícola 2001-2002 INRENA, (2004). Por otra parte, el mayor valor de la $\mathrm{ET}_{\mathrm{a}}$ se encuentra en los espejos de agua ( $5.2 \mathrm{~mm} /$ día), según se ve en la Tabla 3 y la Fig. 4 , pero el volumen de agua consumido es mayor en la vega de ciperáceas $\left(17669 \mathrm{~m}^{3}\right)$ que en los espejos 
de agua (11502 $\left.\mathrm{m}^{3}\right)$, dada la mayor extensión de la primera, y a que no presente estrés hídrico durante el año.

Tabla 3. Resultados de la $E T_{a}$ estimados por SEBAL, para las coberturas del suelo en el humedal Paraíso.

\begin{tabular}{|l|l|l|l|l|c|}
\hline $\begin{array}{l}\text { Cobertura } \\
\text { suelo }\end{array}$ & $\begin{array}{c}\text { Área } \\
\text { (ha) }\end{array}$ & $\begin{array}{c}\text { Rango ET } \\
\text { (mm/día) }\end{array}$ & $\begin{array}{c}\mathbf{E T}_{\mathbf{a}} \\
\text { (mm/día) }\end{array}$ & STD & $\begin{array}{c}\mathbf{E T}_{\mathbf{a}} \\
\text { (m) })\end{array}$ \\
\hline $\begin{array}{l}\text { Espejos de } \\
\text { agua }\end{array}$ & 221.2 & $4.3-6.1$ & 5.2 & 0.16 & 11502 \\
\hline $\begin{array}{l}\text { Vega } \\
\text { ciperaceas }\end{array}$ & 384.1 & $3.4-4.9$ & 4.6 & 0.23 & 17669 \\
\hline
\end{tabular}

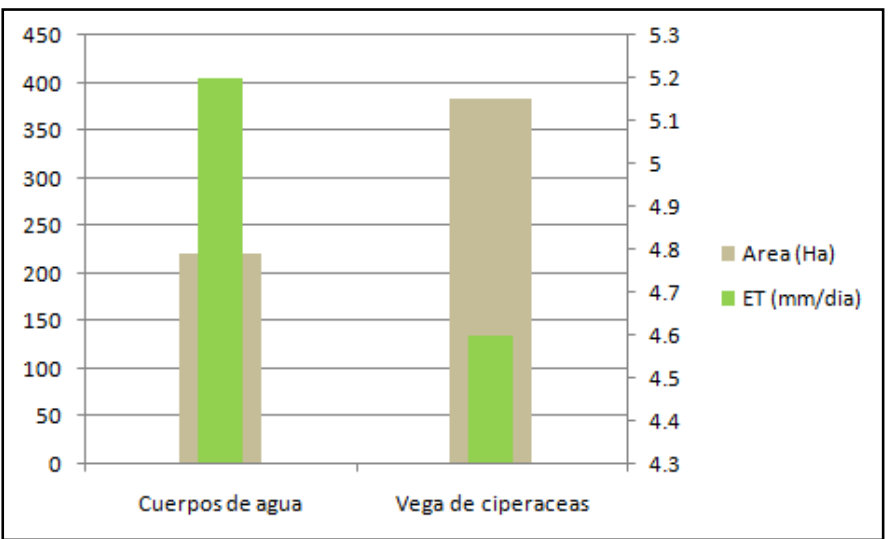

Figura 4: $\mathrm{ET}_{\mathrm{a}}$ en el humedal Paraíso. La mayor $\mathrm{ET}_{\mathrm{a}}$ se da en los espejos de agua, pero por su menor extensión, el mayor consumo de agua se da en la vega de ciperáceas.

En la Figura 5, se puede apreciar la variación espacial de la $\mathrm{ET}_{\mathrm{a}}$ dentro del humedal. En esta imagen se aprecian claramente algunos patrones de la $\mathrm{ET}_{\mathrm{a}}$ debido a la existencia de diferentes hábitats en el humedal, los cuales se comportan de manera distinta antes los intercambios de energía con la atmosfera. En la parte central del humedal, se destaca la vega de ciperáceas con una diferencia en la evapotranspiración debido a los distintos tipos de vegetación que la conforman. En la orilla ubicada al este de la vegetación del humedal, la $\mathrm{ET}_{\mathrm{a}}$ es menor debido a la presencia de gramadales, mientras que el lado puesto la $\mathrm{ET}_{a}$ es mayor por la presencia de los juncales. Las lagunas presentan algunas diferencias en la $\mathrm{ET}_{\mathrm{a}}$, debido a las diferencias en turbidez y a la presencia de vegetación flotante y sumergida. También se aprecian algunas zonas de baja $E_{a}$ dentro del humedal, las cuales corresponden a suelos salinos y suelos $\sin$ vegetación.

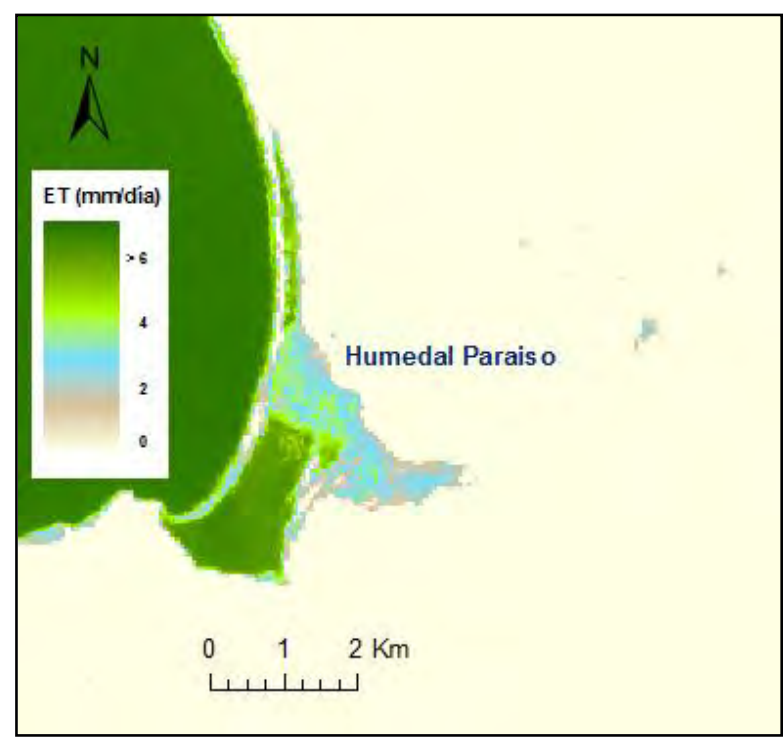

Figura 5: Mapa de la $\mathrm{ET}_{\mathrm{a}}$ para el humedal Paraíso. Se aprecia la diferencia de patrones debido a la distintos ambientes y estados de la vegetación para el día 08/04/2000

El mapa final de evapotranspiración estimado por SEBAL, se ve influenciado en gran medida por la resolución de la banda térmica Algunas parcelas agrícolas no son detectadas por su escaza extensión, lo que modifica el valor de la temperatura estimado por el sensor y por ende, los componentes del balance de energía. La resolución del pixel en la banda 6 es de $60 \mathrm{~m}$ en el Landsat 7 y es de $120 \mathrm{~m}$ en el Landsat 5, ambos actualmente se encuentran operativos, pero existe una necesidad por mejorar esta resolución para obtener un mayor detalle en la estimación de la evapotranspiración a escalas mayores.

El factor de nubosidad es otra limitante en la aplicación de SEBAL. Las nubes se deberán detectar y enmascarar al momento de aplicar el modelo, ya que por su baja temperatura, ocasiona resultados erróneos en la estimación de la evapotranspiración debajo de esta. Igualmente el tipo de suelo, rocas y las sombras producen algunos resultados erróneos, en las zonas desérticas, lo que puede ser evitado con un mapa de usos y coberturas del suelo.

La presencia de corrientes advectivas, también pueden influir en la estimación final de la evapotranspiración, sobre todo en horas de la tarde en que la velocidad del viento es mayor, lo que podría corregirse si se cuenta con datos meteorológicos horarios medidos en el humedal (Tasumi, 2003). 
Los resultados obtenidos en esta primera aplicación de SEBAL se consideran aceptables, ya que están dentro de los limites esperados para la estimación de la $\mathrm{ET}_{\mathrm{a}}$ diaria por medio del modelo SEBAL, según Bastiaanssen, et al. (2005), no menor de $85 \%$ a escala diaria. Se espera que con mejores datos meteorológicos e información medida in situ en el humedal, el modelo SEBAL calcule con mayor precisión la $\mathrm{ET}_{\mathrm{a}}$ en el humedal.

\section{CONCLUSIONES}

En la conservación de los humedales costeros del Perú, es básico el conocimiento de las condiciones ecohidrológicas de este tipo de ecosistema. Al ser el humedal Paraíso dependiente de los excedentes hídricos de la cuenca, un cambio en las aportes de agua en el humedal podría modificar las funciones y servicios ambientales que brinda este ecosistema. Por lo tanto, un conocimiento de su demanda hídrica es crítico para su conservación.

En este trabajo se demuestra la factibilidad de aplicar el modelo SEBAL y las imágenes del satélite Landsat, para estimar espacialmente la evapotranspiración actual del humedal para una fecha específica, con la ventaja de que sólo se necesita información meteorológica de rutina y que las imágenes de satélite empleadas son de uso gratuito.

Los espejos de agua del humedal tuvieron una $\mathrm{ET}_{\mathrm{a}}$ media de $5.2 \mathrm{~mm} / \mathrm{día}$ con un volumen evapotranspirado de $11,502 \mathrm{~m}^{3}$, la vegetación del humedal (vega de ciperáceas) tuvo una $\mathrm{ET}_{a}$ de 4.6 $\mathrm{mm}$ /día con un volumen evapotranspirado de 17,669 $\mathrm{m}^{3}$. Dada la mayor extensión de la vegetación del humedal, esta consume mayor cantidad de agua que los cuerpos de aguas sur y norte juntos.

La comparación de los valores estimados por SEBAL con los obtenidos usando la fórmula FAO Penman-Monteith, incluyendo el empleo del coeficiente de cultivo (Kc), aunque no son absolutos, muestran una buena concordancia en los valores de $\mathrm{ET}_{\mathrm{a}}$ y la $\mathrm{ET}_{\mathrm{c}}$ estimados en la vegetación del humedal y en las áreas agrícolas del valle, con un error medio menor del 10\%. La aplicación de este modelo debería de validarse con mediciones in situ, como el uso de la bandeja de evaporación, o técnicas micrometeorológicas, como la razón de Bowen o covarianza de remolinos como se ha hecho en otros países donde se ha aplicado SEBAL. Sin embargo, se puede decir en una primera aproximación que los resultados del modelo son aceptables en la estimación espacial de la $\mathrm{ET}_{\mathrm{a}}$.

La presencia de nubes es un factor que limita la aplicación de SEBAL. Las imágenes captadas por los satélites Landsat 5 y 7 son cada 16 días por lo que existe pocas oportunidades de conseguir imágenes libres de nubosidad. Se debería complementar las imágenes Landsat con imágenes de mayor resolución temporal como MODIS (imágenes diarias).

La falta de información meteorológica horaria limita un poco la obtención de valores más precisos de la evapotranspiración con SEBAL. Igualmente la falta de algunos datos diarios condicionan los resultados. Si bien, la estimación de la $\mathrm{ET}_{\mathrm{a}}$ diaria en el humedal Paraíso es importante, para fines de gestión del agua en el humedal es necesario estimar la $\mathrm{ET}_{\mathrm{a}}$ en periodos de tiempo mayores (anuales), lo que se puede lograr con el uso de escenas multitemporales.

\section{AGRADECIMIENTOS}

Queremos agradecer al personal profesional y técnico de la Administración Local de Agua Huara, por las facilidades logísticas prestadas en la realización del presente trabajo. Igualmente, a la Oficina del Sistema Nacional de Información de Recursos Hídricos de la Autoridad Nacional del Agua por la información meteorológica utilizada en la aplicación del modelo SEBAL y a la Interface de Datos para las Ciencias de la Tierra (ESDI) de la Universidad de Maryland por la imagen de satélite usada.

\section{REFERENCIAS}

[1] Ahmad, M., Thulani, M., Love, D., Kongo, V., Mul, M., Kinoti, M y J. (2005) Estimating actual evapotranspiration through remote sensing techniques to improve agricultural water management: a case study in the transboundary Olifants catchment in the Limpopo basin, South Africa . 6th WaterNet/WARFSA/GWP Annual Symposium, 1-4 November 2005, Ezulwini , Swaziland.

[2] Allen, R. G., Pereira, L. S., Raes, D. y Smith, M. (1998) Crop evapotranspiration, guidelines for computing crop water requirements, FAO Irrig. and Drain. Pap. 56, 300 pp., Food and Agric. Organ. of 
the U. N. (FAO), Rome, Italy.

[3] Allen, R.G. (1998) Predicting evapotranspiration demands for wetlands. ASCE Wetlands Engineering and River Restoration Conference, Denver Colorado March 20-29.

[4] Allen, R.G., Bastiaanssen, W.G.M., Tasumi, M. y Morse, A. (2001) Evapotranspiration on the watershed scale using the SEBAL model and Landsat Images. Paper \# 01-2224, ASAE, Annual International Meeting; Sacramento, CA.

[5] Bastiaanssen, W.G.M., Menenti, M., Feddes, R.A. y Holtslag, A.A.M. (1998a) A remote sensing surface energy balance algorithm for land (SEBAL): Part 1. Formulation. J.Hydrol., 212-213, pp. 198212.

[6] Bastiaanssen, W.G.M., Pelgrum, H., Wang, J., Ma, Y., Moreno, J., Roerink, G.J. y Van derWal, T. (1998b) The Surface Energy Balance Algorithm for Land (SEBAL): Part 2 Validation, J. of Hydrology, 212-213: 213-229.

[7] Bastiaanssen, W. G. M. (2000) SEBAL-based sensible and latent heat fluxes in the irrigated Gediz Basin, Turkey.. Journal of Hydrology, 222, pp. $87-100$.

[8] Bastiaanssen, W.G.M., Noordman, E.J.M., Pelgrum, H., Davids, G., Thoreson B.P. y Allen, R.G. (2005) SEBAL model with remotely sensed data to improve water resources management under actual field conditions. J. Irrig. and Drain. Engrg, ASCE 131(1):85-93.

[9] Hafeez, M.M., Chemin, Y., Van De Giesen, N. y Bouman, B. (2002) Field Evapotranspiration estimation in central Luzon, Philippines using different sensors: Landsat 7 ETM+, Terra Modis and Aster. In: ISPRS/CIG conference July 8-12, Canada.

[10] INRENA (2004) Propuesta de asignaciones de agua en bloque - volúmenes anuales y mensuales para la formalización de derechos de uso de agua en el valle huaura. PROFODUA-IRH.

[11] Jia, L., Xi, G., Liu, S., Huang, C., Yan, Y., y Liu, G. (2009) Regional estimation of daily to annual regional evapotranspiration with MODIS data in the Yellow River Delta wetland, Hydrol. Earth Syst. Sci., 13, 1775-1787.

[12] Kustas, W. P. (1990) Estimates of evapotranspiration with a one- and two-layer model of heat transfer over partial canopy cover.. Journal of Applied Meteorology, 29, pp. 704-715.

E-mail: egarciag@ana.gob.pe
[13] Mohamed, Y.A., Bastiaanssen, W.G.M. y Savenije, H.H.G. (2004) Spatial variability of evaporation and moisture storage in the swamps of the upper Nile studied by remote sensing techniques', Journal of Hydrology, Vol. 289, pp. 145-164.

[14] MINAG (2008) Delimitación y codificación de unidades hidrográficas del Perú. Resumen ejecutivo pp. 36.

[15] Morse, A., Tasumi, M., Allen, R. G. y Kramber, W. J. (2000) Application of the SEBAL methodology for estimating consumptive use of water and streamflow depletion in the Bear river basin of Idaho through remote sensing - Final Report. Idaho Department of Water Resources - University of Idaho.

[16] Oberg, J.M. y Melesse, A. M. (2006) Evapotranspiration Dynamics at an Ecohydrological Restoration Site: An Energy Balance and Remote Sensing Approach. Journal of the American Water Resources Association, vol. 42, Issue 3, p.565-58.

[17] Tasumi, M., Bastiaanssen, W.G.M. y Allen, R.G. (2000) Application of the SEBAL methodology for estimating consumptive use of water and stream flow depletion in the Bear River Basin of Idaho through remote sensing. EOSDIS Project Final Report, Appendix C.

[18] Tasumi, M. (2003) Progress in operational estimation of reginal evapotranspiration using satellite imagery. Ph.D. Dissetation, University of Idaho, Moscow, ID.

[19] Thomthwaite, C.W. y Mather, J.R. (1955) The water balance. Publications in climatology. Centerton, NJ: Drexel Institute of Technology. Vol. VIII, No. 1

[20] Waters, R., Allen, R., Tasumi, M., Trezza, R. y Bastiaanssen, W.G.M. (2002) SEBAL (Surface Energy Balance Algorithms for Land): Advanced Training and Users Manual. Idaho Department of Water Resources. p. 98. 